J. Lake Sci.(湖泊科学), 2008, 20(2): 215-220

http://www.jlakes.org. E-mail: jlakes@niglas.ac.cn

(C)2008 by Journal of Lake Sciences

\title{
工具种轮叶黑藻的组织培养与快速繁殖
}

\author{
蒋金辉 ${ }^{1,2,3}$, 周长芳 ${ }^{1,2}$, 安树青 ${ }^{1,2^{* *}}$, 关保华 ${ }^{2}$, 蔡 颖 $^{2}$ \\ (1: 南京大学生命科学学院, 污染控制与资源化研究国家重点实验室, 南京 210093) \\ (2: 南京大学生命科学学院, 湿地生态研究所, 南京 210093) \\ (3: 中国科学院南京地理与湖泊研究所, 南京 210008)
}

摘 要: 利用组织培养技术对工具种轮叶黑藻进行快繁研究, 建立无菌体系后, 在 0.5 和 1 倍 MS 培养液中添加 $0.5-2 \mathrm{mg} / \mathrm{L} \mathrm{BA}$ 及 0.1-0.2 $\mathrm{mg} / \mathrm{L}$ IAA 进行芽诱导; 在 0.25、0.5 和 1 倍 MS 培养液中添加 $0.1-0.2 \mathrm{mg} / \mathrm{L}$ IAA 进行根诱导. 结果发现, 1 倍 MS+ $1 \mathrm{mg} / \mathrm{L} \mathrm{BA}+0.1 \mathrm{mg} / \mathrm{L}$ IAA 培养基获得最大芽数及芽生物量, 最适合芽诱导; 0.5 倍 $\mathrm{MS}+0.1 \mathrm{mg} / \mathrm{L}$ IAA 或 1 倍 $\mathrm{MS}+0.2 \mathrm{mg} / \mathrm{L}$ IAA 培养基最能促进根的发展. 芽增殖使轮叶黑藻繁殖效率达每季度 1800 倍以上; 再经根诱导, 快速形成完整植株, 提高定居能 力. 在室外水体中以两种密度 $\left(100\right.$ 株 $/ \mathrm{m}^{2}, 320$ 株 $\left./ \mathrm{m}^{2}\right)$, 三种底质(沙, 泥, 泥+沙 $\left.(1: 1 \mathrm{~V} / \mathrm{V})\right)$ 上进行移栽, 成活率 $100 \% .21 \mathrm{~d}$ 后, 高密 度群落覆盖度大于 $70 \%$. 表明该技术不仅可在短期内获得大量种苗，且种质优，为沉水植被恢复工程奠定关键的基础.

关键词: 组织培养; 沉水植被; 大规模扩繁; 湿地恢复

\section{Vast propagation of submerged tool species Hydrilla verticillata with tissue culture method}

\author{
JIANG Jinhui $^{1,2,3}$, ZHOU Changfang ${ }^{1,2}$, AN Shuqing ${ }^{1,2}$, GUAN Baohua ${ }^{2}$ \& CAI Ying ${ }^{2}$ \\ (1: State Key Laboratory of Pollution Control and Resource Uses, School of Life Science, Nanjing University, Nanjing 210093, \\ P.R.China) \\ (2: Institute of Wetland Ecology, School of Life Science, Nanjing University, Nanjing 210093, P.R.China) \\ (3: Nanjing Institute of Geography and Limnology, Chinese Academy of Sciences, Nanjing 210008, P. R. China)
}

Abstract: The vast propagation of Hydrilla verticillata was studied based on the tissue culture technique. After aseptic system was established, half and full strength of Murashige and Skoog-based liquid media(MS), in addition with 0.5-2 mg/L 6-Benzylaminopurine (BA) and 0.1-0.2 mg/L indoleacetic acid (IAA), were used for shoot induction. Meanwhile, quarter, half and full strength of MS plus 0.1-0.2 mg/L IAA were useded for root induction. The results showed that the combination of full strength of MS plus $1 \mathrm{mg} / \mathrm{L}$ BA and $0.1 \mathrm{mg} / \mathrm{L}$ IAA was the best medium for shoot induction because the plants on such combined condition had more shoot number and shoot biomass than those in the other mixtures. Half strength of MS plus $0.1 \mathrm{mg} / \mathrm{L}$ IAA or full strength MS plus $0.2 \mathrm{mg} / \mathrm{L}$ IAA was the most proper media for root induction, and they can greatly promote the development of roots. The propagation was greatly enhanced with the shoot induction in the optimal environment, amounting to 1800 times per quarter. The root induction made these shoots grow into whole plants quickly, which enhanced the colonization ability of propagules at new habitat. In the outdoor experiment, all plants survived at two densities $\left(100\right.$ plants $/ \mathrm{m}^{2}, 320$ plants $\left./ \mathrm{m}^{2}\right)$ on three sediment types (sand, clay, sand + clay $(1: 1$ $\mathrm{V} / \mathrm{V})$ ). After 21 days, the population coverage was more than $70 \%$. The experiments demonstrated that tissue culture was an effective method of propagation of tool species to get the vast number of seedlings, and ensures the quality of the plants, and finally establishes a key basis for restoration engineering of submerged vegetation.

Keywords: Tissue culture; submerged vegetation; vast propagation; wetland restoration

* 科学技术部太湖 “863” 专项(2002AA601012-06)和镇江 “863” 专项(2003AA06011000-04)联合资助. 2007-03-14 收稿; 2007-06-06 收修改稿. 蒋金辉, 男, 1979 年生, 博士研究生; E-mail: chinascrub@gmail.com.

** 通讯作者; E-mail: anshq@nju.edu.cn. 

作为主要的生产者, 沉水植物是湖泊生态系统的重要成员 ${ }^{[1]}$, 对于维持该系统的健康和稳定有着关 键作用 ${ }^{[2-3]}$. 除富集有害重金属以外, 沉水植物还能吸收环境中(特别是水环境中)过多的营养物质, 有效 防治水体富营养化 ${ }^{[4]}$; 减少底泥再悬浮并促进水体中悬浮颗粒物的沉降, 增加水体透明度, 从而支撑湖 泊浮游生物群落以至整个食物链 ${ }^{[-6]}$; 同时, 它也为鱼虾提供㙁料及避难所 ${ }^{[7-8]}$. 目前, 我国湖泊富营养化 问题日益严重, 沉水植被严重退化甚至消失 ${ }^{[9]}$. 前期研究表明, 恢复沉水植被, 是恢复整个水生生态系统, 改善其水质的一个有效方法 ${ }^{[10]}$.

对于长期严重退化的湖泊生态系统, 由于严重缺乏沉水植物种质资源, 其植被恢复多通过人工引种 先锋工具种进行, 即从邻近健康的自然生态系统中收集种源并移植到目标系统中 ${ }^{[1]}$. 然而, 目前能提供 种源的生态系统越来越少. 同时, 沉水植物在收获过程中难度较大, 植物根系破坏严重, 移栽不易成活, 造成种源的浪费, 甚至恢复工程的失败 ${ }^{[12]}$. 而且大量植物的移植, 也会引发一系列生态问题, 不仅会导 致种源生境的破坏, 也有在目标系统中引人不必要的病原菌或人侵种的风险 ${ }^{[11,13]}$. 为此, 本文尝试通过 植物组织培养(包括芽诱导和根诱导)的方式大量扩繁工具种, 并进行其室外存活率及生长测试; 在克服 扞插繁殖时间和场地限制的基础上, 有效增加其繁殖效率和建群能力. 该方法基于沉水植物片段的克隆 繁殖能力 ${ }^{[14]}$, 希望在短期内获得大量健壮植株; 且所获种苗纯净, 不带任何不必要的菌类及杂草, 利于 初期植被的快速建群. 通过本研究, 探索该技术在水体植被恢复中应用的有效性和具体实施方式.

\section{1 实验材料与方法}

\section{1 实验材料}

轮叶黑藻(Hydrilla verticillata) 是水鳖科(Hydrocharitaceae)的一种多年生沉水植物, 普遍生长在我国 各处水域中 ${ }^{[15]}$. 由于其较强的耐污性, 常在富营养化水体沉水植被恢复工程中作为先锋物种 ${ }^{[16]}$. 目前, 轮叶黑藻的繁殖主要通过断枝扦插等方法解决 ${ }^{[17]}$.

从东太湖 $\left(30^{\circ} 55^{\prime} \mathrm{N}, 119^{\circ} 52^{\prime} \mathrm{E}\right)$ 采集轮叶黑藻的健壮芽尖, 在肥㿝水中浸泡约 $2 \mathrm{~h}$, 再在自来水下冲洗 浸泡 $1 \mathrm{~d}$. 取约 $2 \mathrm{~cm}$ 长的芽段, 在无菌操作台上用 $70 \%$ 的乙醇浸泡 $30 \mathrm{~s}$ 及 $10 \%$ 的次氯酸钠表面消毒 $10 \mathrm{~min}$, 用无菌水反复冲洗 5 遍, 转人装有 $40 \mathrm{ml}$ 的 Murashige-Skoog $+3 \%$ 蔗糖(MS, 下同)无菌培养液的 $100 \mathrm{ml}$ 三 角瓶中进行静置培养 ${ }^{[18]}$. 每 2 周更换一次培养液, 8 周后收取实验所需无菌芽段或芽丛. 培养在组培室中 进行, 平均光照 $30 \mu \mathrm{E} /\left(\mathrm{m}^{2} \cdot \mathrm{s}\right)$, 光照周期 $12 \mathrm{~h} / \mathrm{d}$, 室内温度 $(25 \pm 1)^{\circ} \mathrm{C}$.

\section{2 实验方法}

1.2.1 芽诱导实验 为研究不同培养液浓度及激素配比对轮叶黑藻芽诱导的效果, 本研究采用了 0.5 倍和 1 倍 MS 基本培养液, 并添加了 $0.1-0.2 \mathrm{mg} / \mathrm{L}$ 的 IAA 及 $0.5-2 \mathrm{mg} / \mathrm{LBA}$, 以无激素培养液为对照, 共设置了 14 个处理(表 1). 培养基装于 $100 \mathrm{ml}$ 的三角瓶中, 每瓶 $40 \mathrm{ml}$, 每个处理 5 个重复. 培养基采用高压灭菌锅 $120^{\circ} \mathrm{C}$ 灭菌处理 $20 \mathrm{~min}$ 后, 接人大小相似的轮叶黑藻无菌芽丛, 每丛约 5 个小芽, 每个小芽长 $1.5 \mathrm{~cm}$ 左 右, 于组织培养室中静置培养. $30 \mathrm{~d}$ 后记录单丛培养物的总芽数和总芽长度, 计算单芽平均长度, 再经鼓 风烘箱中 $60^{\circ} \mathrm{C}$ 下烘至恒重后称取单丛芽生物量.

1.2.2 根诱导实验 设置 9 组根诱导培养基, 其中基本营养浓度采用了 $0.25 、 0.5$ 和 1 倍 MS, 激素添加 0.1-0.2mg/L IAA, 以不加激素的作为对照(表 2). 培养液分装和灭菌方式同芽诱导实验, 但每个处理 10 次重复, 并接人芽诱导实验所得的大小一致的芽丛, 即每丛 5 个小芽, 每个小芽长约 $1.5 \mathrm{~cm}$, 置于组培室 静置培养 $15 \mathrm{~d}$. 收获所有植株, 记录单丛根数和总根长, 再经鼓风烘箱中 $60^{\circ} \mathrm{C}$ 下 $72 \mathrm{~h}$ 以上, 烘至恒重后称 取单丛根生物量.

1.2 .3 室外生长实验 收集经上述根诱导所得的大小一致的完整幼苗, 初始生物量为 $32.46 \pm 0.48 \mathrm{mg} / \mathrm{plant}$, 在室外人工水体中, 以两种密度 $\left(100\right.$ 株 $/ \mathrm{m}^{2}, 320$ 株 $\left./ \mathrm{m}^{2}\right)$ 栽种在三种底质(沙, 泥, 泥 + 沙 $(\mathrm{V} / \mathrm{V}$ 为 $\left.1: 1)\right)$ 上, 每 个处理 3 次重复. 实验均采用自来水培养. 其中沙为河沙, 泥从高度富营养化的太湖中收集. 河沙中含 $8.3 \mu \mathrm{g} / \mathrm{g}$ 有效氮, $2.5 \mu \mathrm{g} / \mathrm{g}$ 有效磷; 泥中含 $103 \mu \mathrm{g} / \mathrm{g}$ 有效氮, $36 \mu \mathrm{g} / \mathrm{g}$ 有效磷; 而自来水中含 $26.7 \mu \mathrm{M}$ 总氮, 1.6 $\mu \mathrm{M}$ 总磷. $21 \mathrm{~d}$ 后收获, 记录植株成活率; 同时, 通过数码相片中植物与空隙的像素比估算群落盖度, 并从 每个重复组中随机挑选 2 株，即每个处理有 6 株完整植株，小心洗净表面沉积物及根部底质，于鼓风烘箱 
中 $60^{\circ} \mathrm{C}$ 下烘至恒重后记录单丛生物量. 计算单位面积生物量 $=$ 平均每株生物量 $\times$ 单位面积株数, 单株相对 生长速率 $=($ 单株生物量-初始单株生物量 $) /$ 初始单株生物量 $\times 100 \%$.

\section{3 数据处理}

室外生长实验的单位面积生物量、单株生物量, 单株相对生长速率, 芽诱导实验中的单丛芽数、平 均芽长及总芽生物量, 根诱导实验的单丛根数、总根长及根生物量采用 SPSS 中的一元方差分析. 在芽诱 导实验的所有处理之间(14 个处理 $\times$ 每个处理 5 个重复), 单丛芽数和平均芽长, 芽数与总芽生物量之间 采用 Pearson 相关性双尾检验. 所有多重比较均采用 Duncan 法，显著性水平为 0.05 .

\section{2 结果}

\section{1 芽诱导}

研究显示, 1 倍 MS 培养液对轮叶黑藻芽数的诱导普遍优于 0.5 倍 MS 培养液 $(P<0.5)$ (表 1$)$. 在相同培 养液浓度下, 除 $0.5 \mathrm{mg} / \mathrm{L}+0.2 \mathrm{mg} / \mathrm{L}$ 处理组略低于对照组之外, BA 和 IAA 不同浓度的组合均能促进芽数的 增加, 且在 0.5 倍 $\mathrm{MS}$ 下添加 $0.5 \mathrm{mg} / \mathrm{L} \mathrm{BA}+0.1 \mathrm{mg} / \mathrm{L}$ IAA, 以及在 1 倍 $\mathrm{MS}$ 下添加 $1 \mathrm{mg} / \mathrm{LBA}+0.1 \mathrm{mg} / \mathrm{L}$ IAA 都能使芽数显著高于对照 $(P<0.05)$. 在所有处理之间, 单丛平均芽长和芽数呈极显著负相关 $(r=-0.428$, $P<0.01, n=70) .0 .5$ 倍 MS 营养浓度下, 轮叶黑藻的平均芽长优于 1 倍 MS 培养组 $(P<0.05)$, 但多数处理组 均能达到或超过初始培养材料长度 $(1.5 \mathrm{~cm})$. 单丛总芽生物量和芽数在本实验中呈正相关 $(r=0.602, P<0.01$, $n=70)$. 在所有组合中, 1 倍 $\mathrm{MS}+1 \mathrm{mg} / \mathrm{L} \mathrm{BA}+0.1 \mathrm{mg} / \mathrm{L} \mathrm{IAA}$ 最适合芽的诱导, 能获得最大芽数和芽生物量; 且该条件下, 芽长已达到后继培养的需要(大于 $1.5 \mathrm{~cm}$ ).

表 1 芽增殖培养基中单丛芽数、平均芽长及单丛总芽重

Tab.1 Shoot number, mean shoot length and individual total shoot biomass in media for shoot induction

\begin{tabular}{ccccccc}
\hline 培养基号 & $\begin{array}{c}\text { MS } \\
\text { (times })\end{array}$ & $\begin{array}{c}\text { BA } \\
(\mathrm{mg} / \mathrm{L})\end{array}$ & $\begin{array}{c}\text { IAA } \\
(\mathrm{mg} / \mathrm{L})\end{array}$ & $\begin{array}{c}\text { 芽数 }^{*} \\
\text { (individuals })\end{array}$ & $\begin{array}{c}\text { 平均芽长 } \\
(\mathrm{cm})\end{array}$ & $\begin{array}{c}\text { 单丛总芽重 } \\
(\mathrm{mg})\end{array}$ \\
\hline $1(\mathrm{CK})$ & 0.5 & 0 & 0 & $28.6 \pm 1.0^{\mathrm{ef}}$ & $2.2 \pm 0.2^{\mathrm{bc}}$ & $104.7 \pm 9.4^{\mathrm{c}}$ \\
2 & 0.5 & 0.5 & 0.1 & $45.8 \pm 2.1^{\mathrm{bc}}$ & $1.9 \pm 0.1^{\mathrm{de}}$ & $89.3 \pm 4.9^{\mathrm{cd}}$ \\
3 & 0.5 & 1 & 0.1 & $32.8 \pm 2.0^{\mathrm{def}}$ & $2.3 \pm 0.1^{\mathrm{b}}$ & $93.7 \pm 9.3^{\mathrm{c}}$ \\
4 & 0.5 & 2 & 0.1 & $32.4 \pm 3.4^{\mathrm{def}}$ & $2.3 \pm 0.1^{\mathrm{b}}$ & $88.8 \pm 4.8^{\mathrm{cd}}$ \\
5 & 0.5 & 0.5 & 0.2 & $25.4 \pm 0.8^{\mathrm{f}}$ & $3.2 \pm 0.1^{\mathrm{a}}$ & $86.6 \pm 4.8^{\mathrm{cd}}$ \\
6 & 0.5 & 1 & 0.2 & $37.2 \pm 1.8^{\mathrm{cde}}$ & $2.0 \pm 0.1^{\mathrm{cd}}$ & $85.1 \pm 5.6^{\mathrm{cd}}$ \\
7 & 0.5 & 2 & 0.2 & $35.8 \pm 3.4^{\mathrm{cdef}}$ & $2.0 \pm 0.1^{\mathrm{cd}}$ & $111.6 \pm 14.1^{\mathrm{bc}}$ \\
$8(\mathrm{CK})$ & 1 & 0 & 0 & $43.8 \pm 2.1^{\mathrm{bcd}}$ & $1.8 \pm 0.1^{\mathrm{de}}$ & $135.3 \pm 4.8^{\mathrm{ab}}$ \\
9 & 1 & 0.5 & 0.1 & $46.0 \pm 2.6^{\mathrm{bc}}$ & $1.3 \pm 0.0^{\mathrm{g}}$ & $61.5 \pm 3.4^{\mathrm{de}}$ \\
10 & 1 & 1 & 0.1 & $61.0 \pm 5.6^{\mathrm{a}}$ & $1.7 \pm 0.1^{\mathrm{ef}}$ & $147.1 \pm 14.2^{\mathrm{a}}$ \\
11 & 1 & 2 & 0.1 & $49.6 \pm 7.9^{\mathrm{b}}$ & $1.5 \pm 0.0^{\mathrm{f}}$ & $95.8 \pm 21.5^{\mathrm{c}}$ \\
12 & 1 & 0.5 & 0.2 & $33.0 \pm 3.7^{\mathrm{def}}$ & $0.9 \pm 0.0^{\mathrm{h}}$ & $43.3 \pm 5.4^{\mathrm{e}}$ \\
13 & 1 & 1 & 0.2 & $49.0 \pm 3.5^{\mathrm{b}}$ & $1.6 \pm 0.1^{\mathrm{f}}$ & $103.1 \pm 7.0^{\mathrm{c}}$ \\
14 & 1 & 2 & 0.2 & $50.4 \pm 4.0^{\mathrm{b}}$ & $1.8 \pm 0.1^{\mathrm{de}}$ & $112.1 \pm 7.6^{\mathrm{bc}}$ \\
\hline
\end{tabular}

$*$ Mean \pm S.E., $n=5 ; C K$ 表示对照; 各指标中的不同字母(a-i)代表组间存在显著性差异，显著水平 0.05 .

\section{2 根诱导}

在未添加激素条件下, 0.25 和 0.5 倍 MS 在根数和总根长上优于 1 倍 MS, 但根生物量无明显变化 $(P>0.05)$ (表 2). 相同 0.25 或 0.5 倍 MS 营养浓度下, 0.1 和 $0.2 \mathrm{mg} / \mathrm{L} \mathrm{IAA}$ 的添加都有利于根数和总根长 的增加. 其中 0.5 倍 MS 下, 0.1 和 $0.2 \mathrm{mg} / \mathrm{L}$ IAA 的添加都能显著增加总根长 $(P<0.05)$, 并相应增加根生 物量 $(P>0.05)$. 在 1 倍 $\mathrm{MS}$ 时, $0.2 \mathrm{mg} / \mathrm{L} \mathrm{IAA}$ 能显著增加根数、总根长和根生物量 $(P<0.05)$. 最终, 0.5 倍 
$\mathrm{MS}+0.1 \mathrm{mg} / \mathrm{L} \mathrm{IAA}$ 和 1 倍 $\mathrm{MS}+0.2 \mathrm{mg} / \mathrm{L} \mathrm{IAA}$ 培养液均能有效诱导根的生长, 能获得最大的根数及根生 物量.

表 2 根增殖培养基的单丛根数、总根长及单丛总根重

Tab.2 Root number, total root length and individual total root biomass in media for root induction

\begin{tabular}{|c|c|c|c|c|c|}
\hline 培养基号 & $\begin{array}{c}\text { MS } \\
\text { (times) } \\
\end{array}$ & IAA (mg/L) & $\begin{array}{c}\text { 根数 }^{*} \\
\text { (individuals) }\end{array}$ & 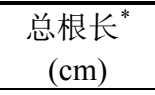 & $\begin{array}{c}\text { 单丛总根重 }^{*} \\
(\mathrm{mg})\end{array}$ \\
\hline $15(\mathrm{CK})$ & 0.25 & 0 & $9.9 \pm 1.2^{\mathrm{b}}$ & $14.2 \pm 1.6^{\mathrm{cd}}$ & $1.0 \pm 0.1^{\mathrm{c}}$ \\
\hline 16 & 0.25 & 0.1 & $12.3 \pm 1.4^{\mathrm{b}}$ & $15.4 \pm 3.2^{\mathrm{cd}}$ & $1.1 \pm 0.3^{\mathrm{c}}$ \\
\hline 17 & 0.25 & 0.2 & $10.2 \pm 1.1^{\mathrm{b}}$ & $23.1 \pm 5.5^{\mathrm{bc}}$ & $1.0 \pm 0.1^{\mathrm{c}}$ \\
\hline $18(\mathrm{CK})$ & 0.5 & 0 & $15.0 \pm 2.2^{\mathrm{ab}}$ & $16.5 \pm 2.1^{\mathrm{cd}}$ & $1.4 \pm 0.2^{\mathrm{bc}}$ \\
\hline 19 & 0.5 & 0.1 & $18.6 \pm 2.0^{\mathrm{a}}$ & $38.5 \pm 6.4^{\mathrm{a}}$ & $2.2 \pm 0.4^{\mathrm{b}}$ \\
\hline 20 & 0.5 & 0.2 & $17.8 \pm 2.6^{\mathrm{a}}$ & $35.5 \pm 7.9^{\mathrm{ab}}$ & $2.3 \pm 0.5^{\mathrm{b}}$ \\
\hline $21(\mathrm{CK})$ & 1 & 0 & $3.1 \pm 0.7^{\mathrm{c}}$ & $3.8 \pm 1.0^{\mathrm{d}}$ & $1.2 \pm 0.4^{\mathrm{c}}$ \\
\hline 22 & 1 & 0.1 & $3.4 \pm 0.4^{\mathrm{c}}$ & $4.1 \pm 0.7^{\mathrm{d}}$ & $0.5 \pm 0.1^{\mathrm{c}}$ \\
\hline 23 & 1 & 0.2 & $18.4 \pm 2.0^{\mathrm{a}}$ & $35.1 \pm 4.2^{\mathrm{ab}}$ & $3.5 \pm 0.4^{\mathrm{a}}$ \\
\hline
\end{tabular}

$*$ Mean \pm S.E., $n=10 ;$ CK 表示对照组; 各指标中的不同字母(a-d)代表组间存在显著性差异, 显著水平 0.05 .

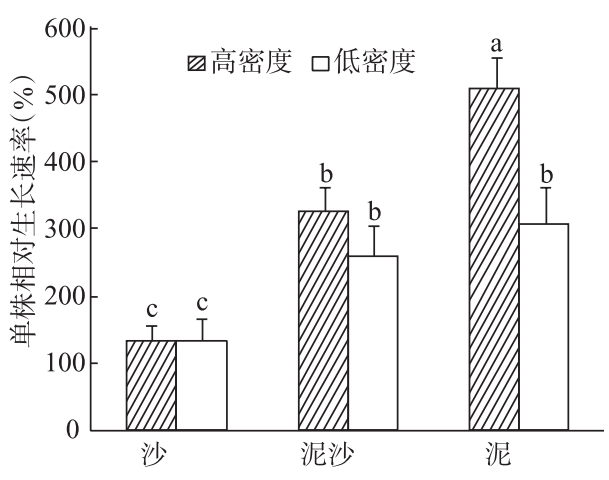

图 1 室外实验各个处理下轮叶黑藻单株相对生 长速率( $\mathrm{a}, \mathrm{b}, \mathrm{c}$ 表示在组间存在显著性差异, 显 著水平 0.05 )

Fig.1 Individual relative growth rate varied among treatments in outdoor experiment

\section{3 室外生长}

三种底质和两种密度种植下, 轮叶黑藻组培苗 的成活率均达到 $100 \%$ (表 3). 相同密度下, 群落的 覆盖度、单株生物量和单位面积生物量均随着基质 中底泥的增加而增加. 三种底质下, 高密度种植下 的群落覆盖度和单位面积生物量分别显著高于低密 度下群落的相应指标 $(P<0.05)$. 其中高密度下轮叶 黑藻在三种底质上覆盖度均达到或超过 $70 \%$. 高密 度种植下植物单株生物量也普遍高于低密度种植下 植物单株生物量, 但仅在泥质基质上差异显著 $(P<0.05)$. 从单株相对生长速率来看, 随底质中泥含 量的增加, 轮叶黑藻的生长速率显著增加 $(P<0.05$, 图 1). 最大相对生长速率出现在泥质底质十高密度 的处理下, 达到 $511.47 \%$.

表 3 室外生长实验的群落指标

Tab.3 Parameters of population in outdoor growth experiment

\begin{tabular}{cccccc}
\hline \multirow{2}{*}{ 底质 } & 密度 & $\begin{array}{c}\text { 成活率 } \\
(\%)\end{array}$ & $\begin{array}{c}\text { 覆盖度 }_{(\%)} \\
\text { 沙 }\end{array}$ & $\begin{array}{c}\text { 单株生物量 }^{*} \\
(\mathrm{mg})\end{array}$ & $\begin{array}{c}\text { 单位面积生物量 }^{*} \\
\left(\mathrm{~g} / \mathrm{m}^{2}\right)\end{array}$ \\
& 高 & 100 & 70 & $76.0 \pm 6.8^{\mathrm{c}}$ & $24.3 \pm 2.2^{\mathrm{c}}$ \\
\multirow{2}{*}{ 泥沙 } & 低 & 100 & 30 & $73.7 \pm 12.8^{\mathrm{c}}$ & $7.4 \pm 1.3^{\mathrm{d}}$ \\
& 高 & 100 & 85 & $139.0 \pm 11.2^{\mathrm{b}}$ & $44.5 \pm 3.6^{\mathrm{b}}$ \\
& 低 & 100 & 50 & $116.2 \pm 15.2^{\mathrm{b}}$ & $11.6 \pm 1.5^{\mathrm{d}}$ \\
& 泥 & 100 & 100 & $198.5 \pm 14.8^{\mathrm{a}}$ & $63.5 \pm 4.8^{\mathrm{a}}$ \\
& 高 & 100 & 75 & $132.1 \pm 18.4^{\mathrm{b}}$ & $13.2 \pm 1.8^{\mathrm{d}}$ \\
\hline
\end{tabular}

* Mean \pm S.E., $n=6$; 各指标中的 a, b, c 代表组间存在显著性差异, 显著水平 0.05 . 


\section{3 讨论及结论}

作为一种克隆植物, 轮叶黑藻的每一个芽, 都可以看作一个繁殖体 ${ }^{[19]}$. 如把芽数看作克隆繁殖指标, 而芽长作为营养生长指标, 本实验中, 轮叶黑藻芽数和平均芽长之间存在显著负相关, 表明该植物为更 有效利用环境资源, 通过协调克隆繁殖和营养生长的相对比例, 改变了资源配置方式. 通过改变资源配 置方式利用环境资源的植物特性在之前文献中就有报导 ${ }^{[20]}$. 本实验中, 在保证作为繁殖体所需的基本芽 长 $(1.5 \mathrm{~cm})$ 下, 兼顾繁殖速率(芽数)和质量(平均芽长及单丛总芽生物量), 认为 1 倍 $\mathrm{MS}$ 添加 $1 \mathrm{mg} / \mathrm{L} \mathrm{BA}+$ $0.1 \mathrm{mg} / \mathrm{L}$ IAA 的培养条件是轮叶黑藻最适宜的扩繁培养基.

自然环境中, 营养浓度的减小会刺激植物增加对根的投入 ${ }^{[21]}$. 在组织培养条件下, 也有报导认为稀 释的 MS 培养液更适合根的诱导 ${ }^{[22]}$. 本实验中, 在缺少激素情况下, 0.5 倍 MS 培养液中轮叶黑藻根系发 育要好于 1 倍 MS, 这表明植物确实通过增加根系发展来补偿周围环境中营养浓度降低可能造成的生长 限制. 但实验中, 0.25 倍 MS 中的根系发育差于 0.5 倍的 MS, 说明这种补偿作用也是有限的. 植物本身 正常的营养生长需要环境资源的支持, 当进一步降低环境营养浓度时, 根系发展同样也受限. 激素的添 加改变了根系在各个培养液中的发育, 且在高浓度的 MS 下, 激素的正向刺激作用更显著.

虽然轮叶黑藻的克隆片段在自然环境中也能够生长繁殖 ${ }^{[19]}$, 但根系的发展是植物在新生境中定植 的必要条件 ${ }^{[23]}$. 因此在人工植被恢复工程中, 发达的根系对植被的快速建群显然是极为有利的. 利用组 织培养技术进行芽和根的诱导, 轮叶黑藻每一个单芽都能发育成多个完整植株. 用新增芽数代表克隆繁 殖效率, 利用实验中篮选所得的培养液, 轮叶黑藻每经一代(30d)就可扩增 12 倍以上, 且扩增倍率呈指数增 长, 即经过一个季度的培养, 就可增殖 1800 倍以上, 远远超出了自然环境中通过枝插快速繁殖每年 10-50 倍的速度 ${ }^{[1]]}$. 且底质和密度种植实验表明, 通过该技术获得的种苗, 具有很强的活力, 可以在室外水体 中生存并迅速建群.

由于组培技术的扩繁效率极高, 被认为是克服自然物种资源逐渐贵乏的有效手段 ${ }^{[24]}$. 组织培养还可 以保证植物材料的一致性, 配合物种选育, 可获得具有特定性状的优良品系 ${ }^{[25]}$. 在水生植被恢复工程中, 由于自然种源收集难度大, 该技术显得更加有利 ${ }^{[26-27]}$. 同时该技术也不受季节限制, 利于植物的科学研究.

室外移栽实验中, 轮叶黑藻在高密度种植情况下的生长速率高于低密度, 这与普遍认为的密度抑制 生长理论不符 ${ }^{[28]}$. 因轮叶黑藻是一种能形成冠层的植物 ${ }^{[29]}$, 作者推测, 较高的密度更有利于其群落的发 展, 即在植被建群初期, 植株之间可通过互利合作, 促进个体生长, 但该推论仍需要进一步实验证明.

总之, 利用组织培养技术, 即先通过 1 倍 $\mathrm{MS}+1 \mathrm{mg} / \mathrm{L} \mathrm{BA}+0.1 \mathrm{IAA} \mathrm{mg} / \mathrm{L}$ 培养液进行芽诱导繁殖, 继 而经 0.5 倍 $\mathrm{MS}+0.1 \mathrm{mg} / \mathrm{L} \mathrm{IAA}$ 或 1 倍 $\mathrm{MS}+0.2 \mathrm{mg} / \mathrm{L} \mathrm{IAA}$ 培养液进行根诱导形成的完整植株, 能在短期内迅 速获得大量轮叶黑藻种苗, 且能在室外 $100 \%$ 成活并迅速建群, 表明该方式可有效满足水体沉水植被恢复工 程对植物工具种苗的要求.

致谢: 非常感谢孙志毅, 钱程等同学在组织培养实验中付出的辛勤劳动, 也感谢杨海波同学在室外实验 中提供的无私帮助.

\section{4 参考文献}

[1] Kufel L, Kufel I. Chara beds acting as nutrient sinks in shallow lakes-a review. Aquat Bot, 2002, 72(3-4): 249-260.

[2] Perrow MR, Meijer ML, Dawidowicz P et al. Biomanipulation in shallow lakes: state of the art. Hydrobiologia, 1997, 342/343: $355-365$.

[3] Vermaat JE, Santamaria L, Roos PJ. Water flow across and sediment trapping in submerged macrophyte beds of contrasting growth form. Archiv Hydrobiol, 2000, 148: 549-562.

[4] Pinder III JE, Hinton TG, Whicker FW. Foliar uptake of cesium from the water column by aquatic macrophytes. $J$ Environ Radio, 2006, 85: 23-47.

[5] Clarke SJ, Wharton G. Sediment nutrient characteristics and aquatic macrophytes in lowland English rivers. Sci Total Environ, 2001, 266: 103-112. 
[6] Kirk KL, Gilbert JJ. Suspended clay and the population dynamics of planktonic rotifers and cladocerans. Ecology, 1990, 71: 1741-1755.

[7] Lauridsen TL, Pedersen LJ, Jeppesen E et al. The importance of macrophyte bed size for cladoceran composition and horizontal migration in shallow lake. J Plankton Res, 1996, 18: 2283-2294.

[8] van Donk E, van de Bund WJ. Impact of submerged macrophytes including charophytes on phyto- and zooplankton communities: allelopathy versus other mechanisms. Aquat Bot, 2002, 72: 61-74.

[9] Seddon S, Connolly RS, Edyvane KS. Large-scale seagrass die back in northern Spencer Gulf, South Australia. Aquat Bot, 2000, 66: $297-310$.

[10] Qiu DR, Wu ZB, Liu BY et al. The restoration of aquatic macrophytes for improving water quality in a hypertrophic shallow lake in Hubei Province, China. Ecol Eng, 2001, 18: 147-156.

[11] Hilt S, Gross EM, Hupfer M et al. Restoration of submerged vegetation in shallow eutrophic lakes- A guideline and state of the art in Germany. Limnologica, 2006, 36: 155-171.

[12] McKinstry MC, Anderson SH. Improving aquatic plant growth using propagules and topsoil in created bentonite wetlands of Wyoming. Ecol Eng, 2003, 21: 175-189.

[13] Brown SC, Bedford BL. Restoration of wetland vegetation with transplanted wetland soil: an experimental study. Wetlands, 1997, 17: 424-437.

[14] Capers RS. Macrophytes colonization in a freshwater tidal wetland (Lyme, CT, USA). Aquat Bot, 2003, 77: $325-338$.

[15] Sutton DL, Latham WGH. Analysis of interstitial water during culture of Hydrilla verticillata with controlled release fertilizers. Aquat Bot, 1996, 54: 1-9.

[16] 李 伟, 刘贵华, 熊秉红等. 1998 年特大洪水后鄱阳湖自然保护区主要湖泊水生植被的恢复. 武汉植物学研究, 2004,22 (4): 301-306.

[17] 连光华, 张圣照. 伊乐藻等水生高等植物的快速营养繁殖技术和栽培方法. 湖泊科学, 1996, 8(增刊): 11-16.

[18] Murashige T, Skoog F. A revised medium for rapid growth and bioassays with tobacco tissue cultures. Physiol Plant, 1962, 15: 473-497.

[19] Hofstra DE, Clayton J, Green JD et al. Competitive performance of Hydrilla verticillata in New Zealand. Aquat Bot, 1999, 63: 305-324.

[20] Chapin FS, Bloom A J, Field CB et al. Plant responses to multiple environment factors. Bioscience, 1987, $37: 49-57$.

[21] Loneragan JF, Asher CJ. Response of plants to phosphate concentration in the solution culture. II. Rate of phosphate absorption and its relation to growth. Soil Sci, 1967, 103: 311-318.

[22] Saxena G, Banerjee S, Rahman L et al. An efficient in vitro procedure for micropropagation and generation of somaclones of rose scented Pelargonium. Plant Sci, 2000, 155: 133-140.

[23] Barrat-Segretain MH, Bornette G, Hering-Vilas-Bôas A. Comparative abilities of vegetative regeneration among aquatic plants growing in disturbed habits. Aquat Bot, 1998, 60: 201-211.

[24] Wang JB, Seliskar DM, Gallagher JL. Growth of tissue culture-regenerated salt marsh monocots in a simulated marsh field plot: Implication for wetland creation and restoration. Ecol Eng, 2007, 29: 8-16.

[25] Seliskar DM, Gallagher JL. Exploiting wild population diversity and somaclonal variation in the salt marsh grass Distichlis spicata (Poaceae) for marsh creation and restoration. Am J Bot, 2000, 87: 141-146.

[26] Huang LC, Chang YH, Chang YL. Rapid in vitro multiplication of the aquatic angiosperm, Anubias barteri var. undulate. Aquat Bot, 1994, 47: 77-83

[27] Zhou CF, An SQ, Jiang JH et al. An in vitro propagation protocol of two submerged macrophytes for lake revegetation in east China. Aquat Bot, 2006, 85: 44-52.

[28] Westoby M. The self-thinning rule. Adv Ecol Res, 1984, 14: 167-227.

[29] Madsen JD, Chambers PA, James WF et al. The interaction between water movement, sediment dynamics and submersed macrophytes. Hydrobiologia, 2001, 444: 71-84. 